\title{
E-ELT, le futur télescope géant européen
}

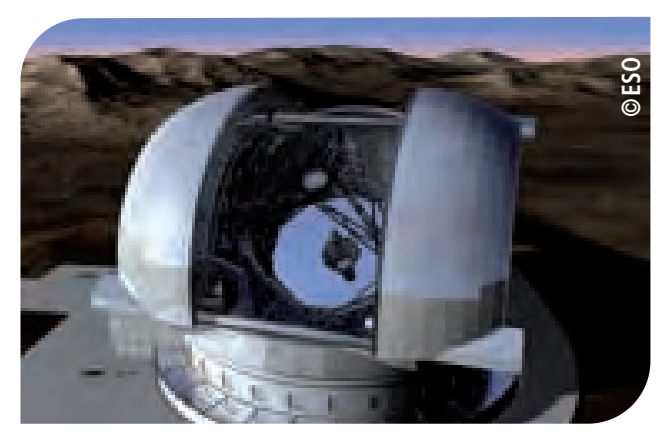

Avec ses $42 \mathrm{~m}$ de diamètre, son système d'optique et son cortège de technologies de pointe, le télescope géant E-ELT (European Extremely Large Telescope) permettra d'atteindre une finesse d'image quatre à cinq fois supérieure à celle des télescopes actuels, tout en offrant une puissance collectrice une vingtaine de fois supérieure. Actuellement en phase de définition avancée, il devrait commencer à nous étonner dès la fin de la décennie, si son lancement est confirmé courant 2011 par les états membres de l'ESO.

\section{Thierry FUSCO \\ Onera \\ Jean-Gabriel CUBY \\ Laboratoire d'Astrophysique de Marseille thierry.fusco@onera.fr}

$\mathbf{S}$ 'il aura fallu attendre près de trois siècles pour que le diamètre des lunettes et télescopes atteigne un mètre, celui-ci a doublé tous les trente ans au cours du vingtième siècle. Et, dans le même temps, les observations du ciel se sont étendues à l'ensemble du spectre électromagnétique, des rayons gamma aux ondes radio. Les plus grands télescopes optiques d'aujourd'hui, construits vers le milieu des années 1990 font entre huit et dix mètres de diamètre. Avec les télescopes géants du futur, les ELTs (pour Extremely Large Telescopes), le diamètre pourrait augmenter d'un facteur trois à quatre en moins de 25 ans.

L'Europe a acquis ses galons en construisant les meilleurs télescopes optiques du moment : les quatre télescopes de huit mètres de diamètre du Very Large Telescope (VLT) installé dans le désert d'Atacama au Chili. Elle pourrait maintenir sa position de leader si son projet de télescope géant de $42 \mathrm{~m}$ de diamètre, I'E-ELT, deux fois plus grand en surface que les projets concurrents américains, était accepté. Ce projet est porté par I'Observatoire européen austral (ESO European Southern Observatory) qui opère le VLT pour la communauté des astronomes de ses quatorze états membres.

L'E-ELT permettra des progrès considérables et décisifs sur de nombreuses questions astrophysiques touchant aux origines de la vie, des planètes, des étoiles, des galaxies et de I'Univers dans son ensemble, en permettant d'investiguer en détail les atmosphères des planètes extrasolaires, de détecter d'éventuelles petites planètes rocheuses comparables à la Terre, de caractériser les premières galaxies aux confins de l'Univers et de comprendre comment ces galaxies ont évolué chimiquement et dynamiquement jusqu'à former celles que nous connaissons aujourd'hui... Mais I'E-ELT $s$ 'attaquera également à des questions de physique fondamentale comme la vitesse d'éloignement des galaxies les unes par rapport aux autres, la potentielle variation des constantes de la physique au cours des âges cosmiques, les lois de la physique autour du trou noir de notre galaxie et des trous noirs supermassifs présents dans de nombreuses galaxies...

\section{Le télescope des extrêmes}

\section{Miroirs}

Pour atteindre tous ces objectifs scientifiques, ce futur géant aura un miroir de $42 \mathrm{~m}$ de diamètre, constitué de $984 \mathrm{seg}$ ments hexagonaux de $1,4 \mathrm{~m}$ de diamètre. Cette surface de verre de plus de 1300 mètres carrés devra épouser parfaitement la forme idéale du miroir, ce qui nécessitera d'aligner les segments les uns par rapport aux autres à quelques dizaines de nanomètres près.

Le reste du train optique du télescope comprendra quatre autres miroirs permettant d'extraire le faisceau optique latéralement vers l'une ou l'autre des plateformes où seront installés les instruments. Parmi ces quatre miroirs, mentionnons le miroir secondaire convexe de plus de $6 \mathrm{~m}$ de diamètre et un miroir adaptatif de plus de $2,5 \mathrm{~m}$ de diamètre déformé en temps réel par plus de 5000 actionneurs pour corriger les aberrations induites par l'atmosphère et les déformations du télescope sous l'effet de la gravité ou des éléments extérieurs. La correction en temps réel des perturbations atmosphériques, appelée optique adaptative, fait ainsi partie intégrante du télescope, au contraire de ses concurrents américains.

\section{Instrumentation}

Dans le plan focal du télescope où se forment les images, sont installés les instruments qui, selon leurs objectifs scientifiques, couvrent des régions différentes de l'espace des paramètres comprenant le champ de vue, le domaine spectral et les résolutions spectrale et spatiale.

L'E-ELT peut potentiellement accueillir une dizaine d'instruments à demeure, principalement aux foyers latéraux du télescope (dits foyers Nasmyth).

La première génération de l'instrumentation de l'E-ELT sera développée sur une dizaine d'années. Deux instruments, dits de première lumière, sont en cours 
de sélection pour équiper le télescope dès sa mise en opération. Huit instruments ont été étudiés pour l'E-ELT, allant de systèmes d'imagerie à multi-longueurs d'onde à des spectrographes ultra précis en passant par des polarimètres, le tout couvrant les

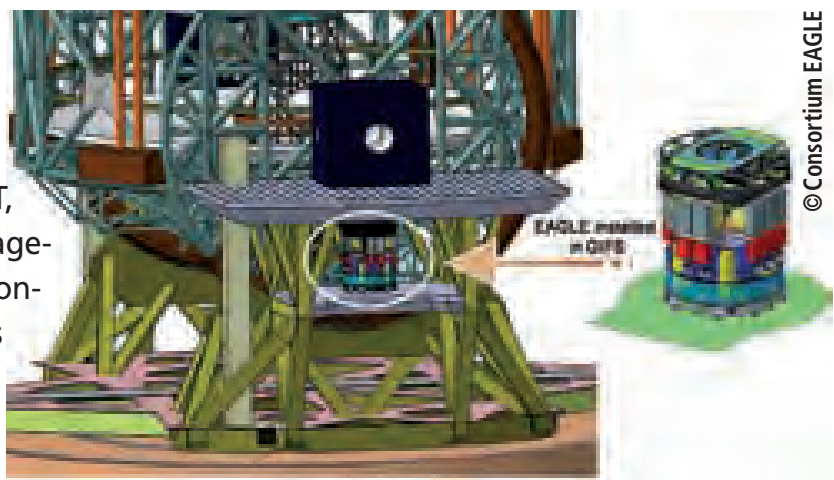
longueurs d'onde du visible et du proche infrarouge (voir complément internet «Liste des instruments scientifiques envisagés pour l'E-ELT»). De plus, deux modules d'optique adaptative feront fonction d'interface entre le télescope et certains instruments.

À titre d'illustration, l'instrument Eagle (figure 1), dont l'étude a été menée en France et en Angleterre, permettra l'observation des premières galaxies dans I'Univers et la spectroscopie bidimensionnelle des galaxies lointaines. Ce système utilisera au mieux la combinaison unique du grand champ et de la haute résolution angulaire qui feront de ce télescope un outil indispensable à l'étude et la compréhension de l'univers.

\section{Structure}

La structure du télescope elle-même est un défi. Elle couvrira plus de 80 mètres au sol et s'élèvera à plus de 60 mètres de haut, pour une masse supérieure à 5000 tonnes. La coupole abritant ce géant (figure 2), de la taille d'un stade de football, doit pouvoir tourner, s'ouvrir et se fermer rapidement et sans encombre par toutes conditions de vent, de température et d'humidité. Elle doit pouvoir

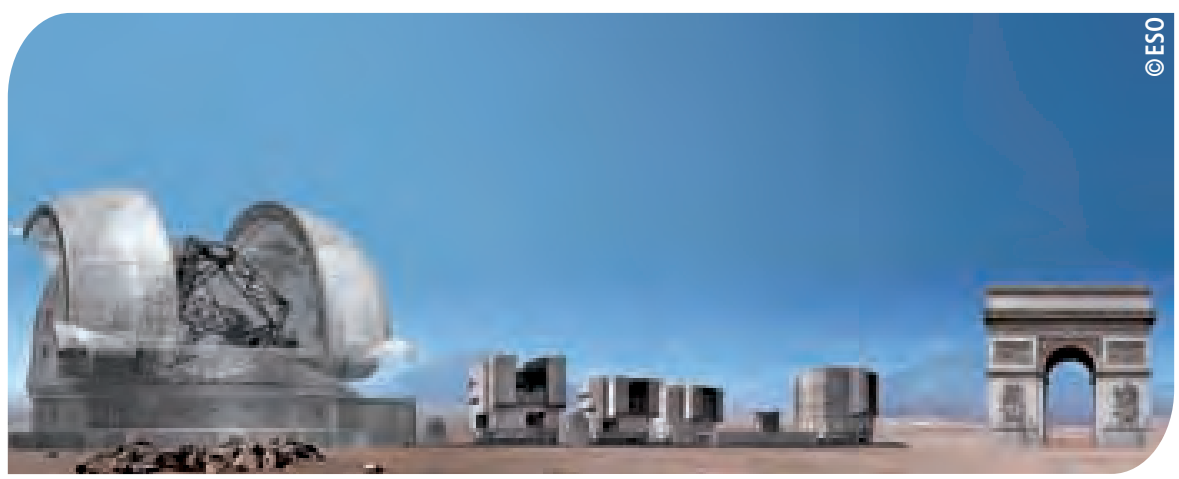

Figure 2. Le future télescope géant européen de $42 \mathrm{~m}$, les quatre télescopes de $8 \mathrm{~m}$ du VLT et I'Arc de triomphe.

Figure 1. L'instrument EAGLE se situera au foyer Nasmyth vertical de l'E-ELT.

survivre à de forts tremblements de terre comme ceux qui ébranlent fréquemment les montagnes du Chili où l'E-ELT sera installé, à tout juste vingt kilomètres du VLT. Cette région, située en plein désert d'Atacama, est l'une des plus arides au monde et des plus propices à l'observation astronomique du fait de son altitude, de sa très faible couverture nuageuse, de sa très faible humidité et de l'exceptionnelle stabilité de son atmosphère.

\section{L'optique adaptative, une technique indispensable à l'E-ELT}

Déjà source de limitation de la qualité $\mathrm{d}$ 'image pour des télescopes de $8 \mathrm{~m}$ de diamètre, les effets perturbateurs de la turbulence atmosphérique sont accrus sur l'E-ELT en étalant la lumière sur une tache focale plusieurs dizaines de fois plus large que la limite de diffraction d'un télescope de $42 \mathrm{~m}$. L'optique adaptative (voir Photoniques $n^{\circ} 33$, février 2008) a pour but de corriger en temps

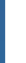


réel ces perturbations et de restituer au télescope son pouvoir de résolution ultime (figure 3). Elle est ainsi au cœur des développements liés à l'E-ELT : le télescope lui-même est adaptatif grâce à un miroir déformable dans son train optique. En plus de ce premier étage de correction intégré au télescope, la plupart des instruments scientifiques actuellement envisagés sera aussi équipée de systèmes de correction adaptative secondaires optimisés en fonction de leurs besoins scientifiques (imagerie haut contraste, correction sur un grand champ...).

Quelles que soient leurs caractéristiques, ces systèmes auront des contraintes et des problématiques communes. Pour la plupart, ils incluront plusieurs miroirs déformables et plusieurs analyseurs pour étendre le champ effectif de correction. De plus, pouvoir disposer de cette correction à chaque observation est essentiel si on veut atteindre les objectifs scientifiques hautement ambitieux visés. Hélas, la lumière provenant de l'objet d'étude étant généralement trop faible pour permettre une mesure de front d'onde pertinente, on utilise une étoile brillante voisine, quand celleci existe, ou plus souvent une étoile guide artificielle, créée par laser, afin de mesurer la distorsion atmosphérique. L'E-ELT utilisera pleinement cette technique en bénéficiant d'au moins six lasers créant une constellation d'étoiles nécessaire à une correction efficace sur un grand champ de vue.

De nombreux prototypes sont déjà en cours de développement en laboratoire ou dans l'industrie afin de prouver la faisabilité technologique des composants clés des systèmes d'optique adaptative requis pour I'E-ELT, notamment: - un laser continu Raman fibré émettant à $589 \mathrm{~nm}$ une puissance d'au moins $50 \mathrm{~W}$;

- un miroir déformable de plus de 2,50 m inclus dans le train optique du télescope. Avec plus de 5000 points d'actionnements permettant des déformations supérieures à 50 microns à une fréquence de plus de $1 \mathrm{kHz}$;

- des micro-miroirs déformables (de quel-
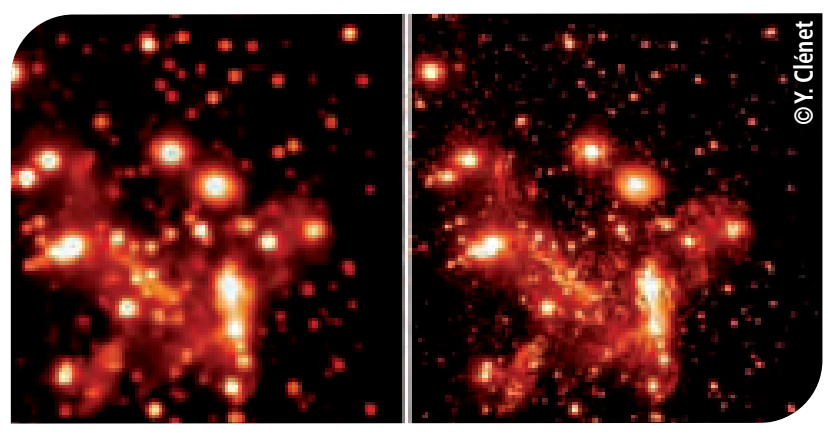

Figure 3. Exemple de résultats d'optique adaptative : sans (a) et avec (b) correction par optique adaptative (le système utilisé est le système NAOS installé sur le Very Large Telescope de I'ESO en 2001).

ques dizaines de centimètres) mais comportant jusqu'à plusieurs dizaines de milliers d'actionneurs ;

- de grandes matrices de détecteurs (jusqu'à 1 mégapixel) très rapides (plusieurs centaines de hertz) et à très faible bruit (moins de quelques électrons de bruit de lecture) pour le visible et le proche infrarouge ;

- des calculateurs temps réel ultrarapides, capables d'absorber le flot d'information nécessaire au contrôle des miroirs déformables.

La combinaison de l'ensemble de ces briques de base permettra à l'E-ELT de disposer de différents concepts d'optiques adaptatives (voir complément internet «Les différents concepts d'optique adaptative de l'E-ELT») s'adaptant aux divers instruments scientifiques et donc aux différents besoins astrophysiques. On pourra ainsi, avec le même télescope, obtenir une gamme de résolution allant de quelques dixièmes de seconde d'arc sur des champs de 100 minutes d'arc carrés au centième de seconde d'arc pour des champs de quelques secondes d'arc carrés et le tout pour près de $100 \%$ du ciel. Enfin, la correction par optique adaptative sera, dans certains cas, poussée à l'extrême (uniquement sur quelques étoiles très brillantes) afin de permettre la détection directe de planètes extrasolaires susceptibles d'abriter d'autres formes de vie.

\section{Un défi décisif pour l'astronomie européenne}

L'observation du ciel et de l'Univers a de tout temps amené son lot de révolutions dans la pensée humaine et dans l'avancement de la science. Avec l'E-ELT, s'il est approuvé, l'Europe se dotera d'une infrastructure de recherche qui permettra d'aborder au cours des prochaines décennies certaines des problématiques les plus ouvertes et les plus intrigantes de l'astrobiologie, de l'astrophysique et de la physique fondamentale. II sera une des pierres angulaires de l'astronomie de la première moitié du XXIe siècle, au côté du télescope spatial James Webb et du projet ALMA de 66 antennes radiomillimétriques en cours de déploiement au Chili, ainsi que de quelques autres grands projets de l'astronomie mondiale, spatiale et au sol.

Ce projet représente un défi technologique, parfaitement maîtrisé, qui exploite le savoir-faire de l'industrie européenne dans le domaine des hautes technologies et le savoir-faire de l'ESO en télescopes optiques et infrarouges. Le succès de ce projet reposera également sur le soutien, l'expérience et le savoir-faire de l'ensemble de la communauté astronomique des états membres de l'ESO, à qui incombera la lourde mais passionnante tâche de réaliser les instruments de ce télescope ainsi qu'une grande partie des systèmes d'optique adaptative, et qui assurera, au final, son exploitation scientifique. Les laboratoires et l'industrie française ont un rôle majeur à jouer dans cette aventure scientifique exceptionnelle.

Compléments internet
- Liste des instruments scientifiques
envisagés pour l'E-ELT avec leurs
caractéristiques principales et leurs
objectifs scientifiques prioritaires.
- Les différents concepts d'optique
adaptative de l'E-ELT.

